\title{
Intraoperative consultation and smear cytology in the diagnosis of brain tumours
}

\author{
Kini JR', Jeyraj $V^{2}$, Jayaprakash $\mathrm{CS}^{3}$, Indira $\mathrm{S}^{3}$, Naik $\mathrm{CNR}^{4}$ \\ ${ }^{1}$ Associate Professor, Kasturba Medical College, Mangalore, ${ }^{2}$ Tutor, ${ }^{3}$ Associate Professor, ${ }^{4}$ Professor, Department of \\ Pathology, Fr Muller Medical College, Mangalore, Karnataka, India.
}

\begin{abstract}
Background: Intraoperative smear cytology provides a rapid and reliable intraoperative diagnosis and guidance to the neurosurgeon during surgical resection and lesion targeting. It also helps the surgeon to monitor and modify the approach at surgery.

Objectives: 1) To assess the utility of intraoperative smear cytology and correlate with the final histopathological diagnosis. 2) To describe the cytomorphological features of common brain tumours in smear preparation.

Materials and methods: The material for this study was obtained from 100 consecutive biopsies of central nervous system neoplasms sent for intraoperative consultation. Smears were prepared from the biopsy samples sent in isotonic saline for immediate processing. The smears were stained by the rapid Haematoxylin and Eosin method. The cytomorphological features were noted and correlated with paraffin section findings.

Results: Of the total 100 cases, 86 showed accuracy when compared with histopathological diagnosis. This was comparable with other studies. Of the remaining, two cases were frank errors, 12 cases showed partial correlation, with five cases showed incomplete typing of the cell type and seven, discrepancy in grading of tumours. The error percentage was $14 \%$. Correlation with clinical details and radiological findings were helpful in improving the accuracy rate.

Conclusions: Smear technique is a fairly accurate, relatively safe, rapid, simple, easily reproducible and cost effective tool to diagnose brain tumours. Smear cytology is of great value in intraoperative consultation of central nervous system pathology.
\end{abstract}

Key words: Intraoperative consultation, smear cytology, central nervous system neoplasms

\begin{abstract}
Cytological evaluation of smear preparations of central nervous system [CNS] neoplasms for a rapid intraoperative diagnosis of brain biopsy samples is now well established and used in many neurosurgical units ${ }^{1}$. High resolution and specialized neuroimaging techniques combined with the expanded use of stereotactic biopsies, commonly require that 'definitive' intraoperative diagnosis be rendered on minute and diminutive tissue specimens. The innately soft nature of brain tissue produces poor quality frozen sections. Cytological methods are ideal for a speedy intraoperative diagnosis of stereotactic CNS biopsy samples because the tissue is minimally distorted and none is wasted ${ }^{2,3}$. The current study was undertaken to assess the utility of intraoperative consultations for cytomorphological diagnosis by smear technique and correlate with histopathological diagnosis.
\end{abstract}

\section{Materials and methods}

One hundred consecutive cases of CNS neoplasms sent for intraoperative consultation to the department of Pathology, Fr. Muller Medical College Hospital, during a period of three years, from March 2003 to February 2006 were studied. The biopsy samples obtained at the time of surgery were transported immediately to the laboratory in small vials containing isotonic saline, for processing. One to two millimeters of the biopsy material were crushed between two glass slides with just enough pressure to spread the tissue into a thin film, fixed in 95\% alcohol and stained by haematoxylin and eosin (H \& E). Paraffin sections were prepared with the residual tissue sample and stained by $\mathrm{H} \&$ E. Relevant clinical and radiological data were noted. Smear cytology diagnoses were correlated with histopathological findings. The tumours were classified according to the World Health Organization classification of CNS neoplasms $2000^{4}$.

\footnotetext{
Correspondence

Dr. Jyoti R Kini

Associate Professor,

Department of Pathology,

Kasturba Medical College,

Mangalore, Karnataka, India

E-mail: kinijyoti@gmail.com
} 
The cases where the intraoperative diagnosis was same as the final diagnosis with an accurate assessment of the cell line and benign versus malignant including the grade were assigned to be in complete correlation. Partial correlation was considered in cases in which \pm 1 grade of deviation in tumour grading was registered or diagnosis of the cell line of origin, in glial neoplasms, was not possible. No correlation was consigned to the cases where the intraoperative diagnosis was not confirmed by the final histological diagnosis.

\section{Results}

Complete correlation between intraoperative cytological and the final histopathological diagnosis was achieved in $86 \%$ of the cases. Diagnostic accuracy increased when cases of partial correlation mainly due to grading deviation were included. The histological and cytological diagnosis of all the cases studied is shown in Table 1.

A significant drop in diagnostic accuracy was observed in oligodendrogliomas, distinguishing between pure astrocytoma/ oligodendroglioma / mixed glioma and the grading of gliomas. Table 2 details the 14 cases misinterpreted on cytological examination. Two cases were frank errors and 12 cases showed partial correlation, comprising seven cases of discrepancy in grading and five where one of the components had been missed during intraoperative consultation. The most common tumours in our series were meningiomas $(25 \%)$. These tumours were relatively easy to smear and showed variable cellularity, high in syncytial type, low in fibrous type. The cells were fairly uniform, arranged in syncytial clusters or in whorls, with eosinophilic cytoplasm and poorly defined boundaries (Fig.1). The nuclei were oval with delicate chromatin and presence of intranuclear inclusions in some. Psammoma bodies were noted in some cases.

Glioblastoma accounted for $17 \%$ of the cases. The tumours spread fairly evenly on smearing and were highly cellular with discohesive cells with sparse cytoplasmic processes (Fig. 2). Marked pleomorphism, atypia, multinucleation, mitotic figures and necrosis were noted along with the characteristic findings of marked endothelial proliferation and glomeruloid bodies.

Astrocytomas contributed to $14 \%$ of the cases. These moderately cellular neoplasms showed some degree of pleomorphism, nuclei with finely or coarsely granular chromatin, inconspicuous nucleoli, variable cytoplasmic processes and fibrillary background. Anaplastic type had in addition increased blood vessels and mitotic figures.

Oligodendrogliomas smear with ease appearing as moderately to highly cellular smears made up of discohesive, relatively small tumour cells with scanty, wispy cytoplasm and uniform round slightly dark nuclei with inconspicuous nucleoli. Background was finely granular and lacked the fibrillarity of other gliomas. Thin walled vessels were prominent and showed foci of calcification.

Schwannomas comprised $13 \%$ of the cases and were difficult to smear due to cohesive cells giving rise to thick smears with three-dimensional twisted rope appearance. The tumour cells were spindle shaped with elongated, wavy nuclei. Verocay bodies and palisading were observed in some smears.

Medulloblastomas (4\%) spread easily and evenly. The smears were densely cellular composed of small cells devoid of identifiable cytoplasm but with round, hyperchromatic nuclei, coarse punctuate chromatin, mitoses and individual cell necrosis.

Pituitary adenomas (4\%) provided cellular smears of closely packed monomorphic cells with round nuclei, finely dispersed chromatin, single small nucleoli and acidophilic to faintly basophilic cytoplasm. Occasional multinucleation was observed but there were no mitoses, necrosis, pleomorphism or cellular processes.

The three cases of craniopharyngiomas were resistant to smearing, revealed cohesive sheets of squamous cells and keratinous debris. Background showed cholesterol crystals, calcified debris, foreign body type giant cells and histiocytes.

Ependymomas represented $2 \%$ of our series and were cellular tumours smeared in cohesive clusters, tissue fragments and sheets of unevenly spread single cells. Perivascular pseudorosettes were prominent. The cells had scanty fibrillary cytoplasm and uniform, bland, round to oval nuclei with dense, finely distributed chromatin and inconspicuous nucleoli.

In one case of choroid plexus papilloma, cytology clearly captured the large papillary tissue fragments and monolayered sheets of singly dispersed tumour cells. The cells had ill defined, pale, scanty cytoplasm, round to oval nuclei with finely granular chromatin and inconspicuous nucleoli. There was no atypia, mitosis or necrosis.

The single case of chordoma smeared easily and was evidenced cytologically by neoplastic cells arranged singly, in aggregates and in tissue fragments in a myxoid background. The cells had voluminous, bubbly eosinophilic cytoplasm, low nuclear to cytoplasmic ratio and ovoid nucleus. Binucleation was also noted in some cells. 
A rare case of meningeal melanocytoma was diagnosed cytologically based on non-cohesive sheets and perivascular aggregates of uniform population of round to polygonal neoplastic cells with round to oval bland nuclei and inconspicuous nucleoli. Cytoplasmic brown pigment was seen within tumour cells and macrophages.

Smears of the one case of lymphoma were highly cellular made up of non-cohesive, monotonous population of lymphoid cells with round and well-defined nuclei, scanty cytoplasm and absence of cytoplasmic processes.

Only one case of metastatic carcinoma was encountered in our study and on cytological analysis demonstrated an easily smeared neoplasm made up of pleomorphic cells with high $\mathrm{N} / \mathrm{C}$ ratio and mitotic activity in cohesive clusters encountered abruptly adjacent to normal brain tissue.

Table 1: Correlation of Histopathological Versus Cytological Diagnosis

\begin{tabular}{|l|c|c|c|}
\hline HPE Diagnosis & Total number & $\begin{array}{c}\text { Correct cytological } \\
\text { diagnosis }\end{array}$ & $\begin{array}{c}\text { Accuracy } \\
\text { (\%) }\end{array}$ \\
\hline Astrocytoma grade I,II,III & 14 & 14 & 100 \\
\hline Glioblastoma multiforme & 17 & 11 & 64.7 \\
\hline Oligodendroglioma \& mixed glioma & 9 & 2 & 100 \\
\hline Ependymoma & 2 & 1 & 100 \\
\hline Choroid plexus papilloma & 1 & 4 & 100 \\
\hline Medulloblastoma & 4 & 25 & 100 \\
\hline Meningioma & 25 & 1 & 100 \\
\hline Melanocytoma & 1 & 13 & 100 \\
\hline Schwannomas /acoustic neuroma & 13 & 4 & 100 \\
\hline Pituitary adenoma & 4 & 3 & 100 \\
\hline Craniopharyngioma & 3 & 1 & 100 \\
\hline Lymphoma & 1 & 0 & 0 \\
\hline Tuberculoma & 1 & 1 & 100 \\
\hline Dermoid cyst & 1 & 1 & 100 \\
\hline Epidermoid cyst & 1 & 1 & 100 \\
\hline Chordoma & 1 & 1 & 100 \\
\hline Eosinophilic granuloma & 1 & & 100 \\
\hline Metastasis & 100 & & \\
\hline Total & & 1 & \\
\hline
\end{tabular}

Table 2: Cases misinterpreted in smear cytology

\begin{tabular}{|l|c|c|c|}
\hline \multirow{2}{*}{ HPE Diagnosis } & Total no of cases & Smear diagnosis & $\begin{array}{c}\text { No. of cases misinterpreted } \\
\text { on cytology }\end{array}$ \\
\hline TB granuloma & 1 & Astrocytoma grade II & 1 \\
\hline $\begin{array}{l}\text { Anaplastic } \\
\text { oligodendroglioma }\end{array}$ & 2 & Anaplastic astrocytoma & 1 \\
\hline Glioblastoma & 17 & Oligodendroglioma grade II & 6 \\
\hline \multirow{2}{*}{ Mixed gliomas } & \multirow{2}{*}{5} & Anaplastic astrocytoma & 4 \\
\cline { 2 - 4 } & & Astrocytoma & 1 \\
\hline
\end{tabular}




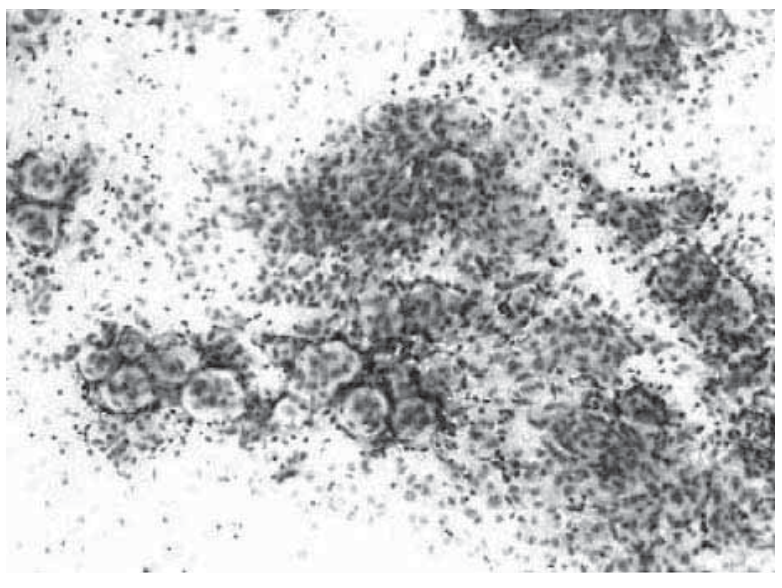

Fig 1: Smear from a meningioma showing whorls and syncytial nests of meningothelial cells. (H \& E, X 100)

\section{Discussion}

Eisenhardt and Cushing introduced intraoperative cytological investigations in the 1920s for rapid examination of neurosurgical specimens and guidance of surgical treatment. The technique can be used to confirm or exclude the presence of neoplasia, to define the tumour cell type and histological grade of the neoplasm.

The practical advantages of smear cytology and intraoperative consultation include the speed and technical ease of preparation, need for minimal technical equipment, and the low expense and of primary significance; the very small sample size required to make the smears does not modify the amount of tissue required for examination of paraffin- embedded sections. As the method does not subject the specimen to a freezing artifact, the chance for a definite, accurate final diagnosis is maximized.

The overall diagnostic accuracy in the present study was $86 \%$. Other workers have also reported diagnostic accuracy varying from $85.4 \%$ to $95.2 \% 0^{1,2,5-10}$. Misinterpretation on cytological evaluation was seen in 14 cases.

No correlation with the final diagnosis was seen in two cases. A case of reactive gliosis secondary to tuberculoma was mistaken for low grade astrocytoma on smear cytology. This was partly a sampling error as the areas diagnostic of tuberculosis had not been picked and the reactive astrocytes were overdiagnosed as neoplastic astrocytes. Other authors ${ }^{1,5,6,8}$ have described similar difficulties in distinguishing between reactive gliosis and low grade astrocytoma especially as both can show similar cytological features. Malignant astrocytes tend to grow along blood vessels and the subarachnoid spaces

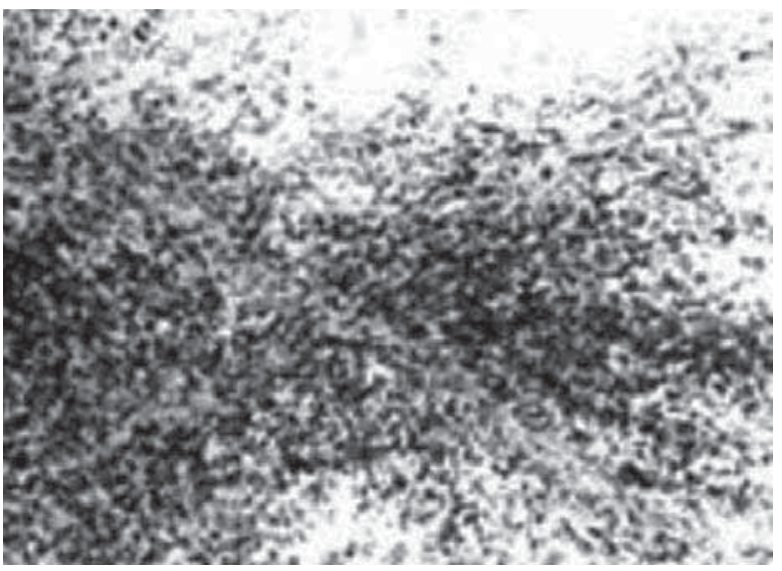

Fig 2: Smear from a glioblastoma showing dense cellularity. (H \& E, X 100)

in some cases. This feature however can be seen only when the architecture is preserved as in paraffin sections. Low grade astrocytomas smear more easily compared to normal parenchyma and reactive processes. On review of the smears, reactive astrocytes had more abundant, evident cytoplasm and more prominent, numerous, long and symmetrical processes. As compared to neoplastic astrocytes, these lack hyperchromatic and lobulated nuclei, progressive atypia and mitoses. Smears of low grade astrocytoma had high cellularity, increased cell dimensions and increased pleomorphism.

An anaplastic oligodendroglioma was mistaken for an anaplastic astrocytoma on smear cytology. Roessler et al have reported similar discrepancies due to lack of uniform appearing nuclei and variable cytoplasmic processes $^{1}$.

Mixed gliomas were also responsible for difficulties in smear interpretation due to predominance of one histological type. The smaller component may be missed especially if they are the less numerous oligodendroglial cells partly due to sampling error but also because of the dense fibrillar background of the astrocytic component ${ }^{5,9}$. Differentiation of astrocytic from oligodendroglial elements is of relevance during stereotactic biopsies due to prompt fixation of these biopsies, reducing the diagnostic oligodendroglial artifacts from formalin fixation, which may lead to a final histogenetic and grade misinterpretation ${ }^{8}$.

Improper grading of astrocytic neoplasms on cytological preparations has been documented by other authors. In fact some consider it inappropriate to attempt to grade CNS neoplasms on biopsy material whether by smear/ frozen technique as astrocytomas are known 
to vary significantly in grade from one area to another within a single tumour. Paraffin sections showed that in the undergraded cases there were areas of both less and more aggressive astrocytomas and the cytological sampling might have failed to show the anaplastic component. Thus small biopsies are unsuitable for grading malignancies ${ }^{5,6,9}$.

Intraoperative consultation is relevant in evaluating the adequacy of the sample often requesting more biopsy when there is disagreement with radiological data or if special investigations like ultrastructural and/ or immunohistochemical studies may be needed where the final diagnosis is expected to be complex.

The newer imaging techniques and approaches have placed the pathologist in a key role in the diagnosis and treatment of patients with CNS neoplasms. It is critical that radiological and clinical data be reviewed before intraoperative evaluation of smear cytology of brain tumours.

\section{Conclusion}

Cytology has emerged as the preferred method for intraoperative diagnosis with the details of cellular morphology defined sharply, avoiding distortion and ice artifacts often introduced by the frozen section technique. Smear cytology is of great value in intraoperative consultation of CNS pathology. Smear technique is a fairly accurate, relatively safe, rapid and simple tool to diagnose brain tumours. Intraoperative smears permit reliable intraoperative guidance during lesion targeting and resection.

\section{References}

1. Roessler K, Dietrich W, Kitz K. High Diagnostic Accuracy of Cytologic Smears of Central Nervous System tumours. A 15- Year experience based on 4172 patients. Acta Cytol 2002;46:667-4.
2. Firlik KS, Martinez AJ, Lunsford LD. Use of cytological preparations for the intraoperative diagnosis of sterotactically obtained brain biopsies: a 19- year experience and survey of neuropathologists. J Neurosurg 1999;91:454-8.

3. Powell SZ. Intraoperative Consultation, Cytologic Preparations and Frozen Section in the Central Nervous System. Arch Pathol Lab Med 2005;129:1635-52.

4. Kleihues P, Cavenee WK, Eds. World Health Organization Classification of Tumours. Pathology and Genetics of Tumours of the Nervous System. Lyon, France: IARC Press; 2000. P.1-4.

5. Cahill EM, Hidvegi DF. Crush Preparations of Lesions of the Central Nervous System. A Useful Adjunct to the Frozen Section. Acta Cytol 1985;29:279-85.

6. Asha T, Shankar SK, Rao TV, Das S. Role of Squash- Smear Technique for Rapid Diagnosis of Neurosurgical Biopsies - A Cytomorphological Evaluation. Indian J Pathol Microbiol 1989;32:152-60.

7. Shah AB, Muzumdar GA, Chitale AR, Bhagwati SN. Squash preparation and frozen section in intraoperative diagnosis of central nervous system tumours. Acta Cytol 1998;42:1149-54.

8. DiStefano D, Scucchi LF, Cosentino L, Bosman C, Vecchinone A. Intraoperative Diagnosis of Nervous System Lesions. Acta Cytol 1998;42:346-56.

9. Torres LFB, Collaco LM. Smear Technique for the Intraoperative Examination of Nervous System Lesions. Acta Cytol 1993;37:34-40.

10. Iqbal M, Shah A, Wani MA, Kirmani A, Ramzan A. Utility of Crush Smear Cytology in Intraoperative Diagnosis of Central Nervous System Lesions. Acta Cytol 2006;50:608-16. 